\title{
Construção de vídeos educativos sobre sofrimento psíquico e cuidado na pandemia do Covid-19: revisão integrativa
}

\author{
Construction of educational videos on psychic suffering and care in the Covid-19 pandemic: \\ integrative review \\ Construcción de vídeos educativos sobre el sufrimiento psíquico y la atención en la pandemia de \\ Covid-19: revisión integradora
}

Recebido: 13/12/2021 | Revisado: 21/12/2021 | Aceito: 22/12/2021 | Publicado: 04/01/2022

\author{
Maria Salete Bessa Jorge \\ ORCID: https://orcid.org/0000-0001-6461-3015 \\ Universidade Estadual do Ceará, Brasil \\ E-mail: maria.salete.jorge @ gmail.com \\ Dina Mara Formiga da Silva \\ ORCID: https://orcid.org/0000-0002-5387-7194 \\ Universidade Estadual do Ceará, Brasil \\ E-mail: dinamara_silva@ hotmail.com \\ José Reginaldo Pinto \\ ORCID: https://orcid.org/0000-0001-8682-7559 \\ Universidade Estadual do Ceará, Brasil \\ E-mail: j.rpinto2018@gmail.com \\ Neyva Torres de Souza Cartaxo \\ ORCID: https://orcid.org/0000-0002-6688-9135 \\ Universidade Estadual do Ceará, Brasil \\ E-mail: neyva.torres@aluno.uece.br
}

\begin{abstract}
Resumo
Utilizar ferramentas seguras, como vídeos educativos institucionais, no intuito de informar melhor sobre a prevenção contra o coronavírus tornou-se uma rotina nas mídias digitais. Esse estudo tem por objetivo resgatar na literatura nacional e internacional publicações de vídeos educativos direcionados para os cuidados em saúde mental para minimizar o sofrimento psíquico ocasionado pela pandemia do covid-19. Desta forma, foi feita uma revisão integrativa, pelo método Prisma, onde foi utilizada a seguinte estratégia de busca: "vídeo" AND "Covid-19" AND "mental health". As buscas dos estudos foram realizadas nos seguintes bancos de dados eletrônicos: Biblioteca Virtual em Saúde (BVS), Science Direct, Web of Science e CINAHL. Restaram 10 estudos para análise após as etapas de refinamento da pesquisa. Os resultados demonstraram que os vídeos educativos promovem apoio social e autoajuda para pessoas de diferentes faixas etárias. Além disso, minimizam a depressão e a ansiedade gerada pelo medo de contrair a infecção, reduz o sentimento de solidão e serve como terapia em saúde mental, além de ser viável, eficaz e acessível para a maioria da população.
\end{abstract}

Palavras-chave: Saúde mental; Vídeos educativos; Covid-19.

\begin{abstract}
Using safe tools, such as institutional educational videos, in order to better inform about the prevention against coronavirus has become a routine in digital media. This study aims to retrieve publications in national and international literature of educational videos aimed at mental health care to minimize the psychological distress caused by the covid-19 pandemic. Thus, an integrative review was carried out, using the Prism method, where the following search strategy was used: "video" AND "Covid-19" AND "mental health". Searches for studies were performed in the following electronic databases: Virtual Health Library (VHL), Science Direct, Web of Science and CINAHL. There were 10 studies left for analysis after the research refinement steps. The results showed that educational videos promote social support and self-help for people of different age groups. In addition, it minimizes depression and anxiety generated by the fear of contracting the infection, reduces the feeling of loneliness and serves as a mental health therapy, in addition to being viable, effective and accessible for the majority of the population.
\end{abstract}

Keywords: Mental health; Educational videos; Covid-19. 


\section{Resumen}

El uso de herramientas seguras, como videos educativos institucionales, para informar mejor sobre la prevención del coronavirus se ha convertido en una rutina en los medios digitales. Este estudio tiene como objetivo rescatar en la literatura nacional e internacional publicaciones de videos educativos dirigidos a la atención de la salud mental para minimizar el sufrimiento psíquico causado por la pandemia de covid-19. Así, se realizó una revisión integradora, utilizando el método Prisma, donde se utilizó la siguiente estrategia de búsqueda: "video" Y "Covid-19" Y "salud mental". Se realizaron búsquedas en los estudios en las siguientes bases de datos electrónicas: Virtual Health Library (VHL), Science Direct, Web of Science y CINAHL. Quedaban 10 estudios para el análisis después de las etapas de refinamiento de la investigación. Los resultados mostraron que los videos educativos promueven el apoyo social y la autoayuda para personas de diferentes grupos de edad. Además, minimizan la depresión y la ansiedad generadas por el miedo a contraer la infección, reducen la sensación de soledad y sirven como terapia de salud mental, y son viables, eficaces y accesibles para la mayoría de la población.

Palabras clave: Salud mental; Videos educativos; Covid-19.

\section{Introdução}

Durante o período da pandemia do Covid-19 foram disponibilizados muitos recursos virtuais para amenizar o sofrimento psíquico da população em vários países. Um desses recursos foi a educação online sobre saúde mental e prevenção da infecção que estiveram disponíveis gratuitamente em várias plataformas.

Em relação ao uso de informações advindas do meio virtual é necessário alertar sobre a disseminação da infodemia, a qual pode intensificar o surgimento de sofrimento psíquico, causando ansiedade, medo excessivo e estresse. A infodemia é caracterizada por notícias sensacionalistas, notícias falsas e divulgação de outras mídias sociais de informações que causam impacto psicológico nas pessoas durante as ondas de disseminação da infecção (Nabuco; Oliveira; Afonso, 2020). A infodemia de informações, se for utilizada de forma indevida, pode causar diferentes reações na população. Ao mesmo tempo que intranquiliza, estimula grupos em estado de vulnerabilidade social aos riscos do sofrimento existencial (Amarante et al., 2020).

Por outro lado, na China, por exemplo, berço da infecção, foram ofertados livros, artigos científicos populares, manuais de psicoeducação e vários vídeos educacionais para o público-alvo de vulnerabilidade psíquica, assim como populações especiais (crianças, mulheres grávidas, idosos, pacientes com transtornos mentais e/ou doenças físicas graves, etc.). A maioria das publicações tinha o intuito de promover a resiliência da saúde mental e acalmar os grupos prioritários que pudessem desencadear o adoecimento mental (Ju et al., 2020).

De acordo com Li et al. (2019), os vídeos educativos de qualidade utilizados pelas autoridades governamentais para atenuar o sofrimento psíquico durante a pandemia ajudam a melhorar a saúde mental das pessoas, pois fornecem um espaço para interações naturais, respostas autênticas e, o mais importante, incorporam expressões que são facilmente esquecidas.

Os vídeos educativos em saúde mental podem ser um apoio externo para orientar a população de como atenuar o sofrimento gerado pelo medo e sentimentos pessimistas aflorados pela Covid-19. No caso do ambiente escolar, Figueiredo et al. (2021) reforça que as crianças necessitam dos seguintes recursos externos para dar continuidade suas atividades cotidianas: (a) disponibilidade de um ambiente online adequado para desenvolverem atividades escolares ( por exemplo, criar plataformas online adequadas), (b) uso de vídeos motivacionais que podem ajudar a melhorar o estilo de vida diário (por exemplo como ter uma dieta balanceada, praticar exercícios em casa e regular o sono), (c) Acesso a informações adequadas (por exemplo, como adotar práticas de higiene corretas) e (d) Acesso a suporte psicológico profissional. Além disso, o apoio social é amplamente entendido como a presença de relações positivas que criam um sentimento de pertencimento, estimulam a confiança e incentivam o autocuidado.

Dispositivos celulares e computadores podem compor ferramentas cruciais de saúde mental durante este período. Seu uso facilita a comunicação com a família e o círculo social, sessões de aconselhamento e informações sobre necessidades 
médicas e psicológicas. O contato direto, com base em ligações para centros de saúde mental, pode rastrear os sintomas de ansiedade e depressão e reduzir o impacto do contexto pandêmico na saúde mental dos mais vulneráveis (Grolli et al., 2020).

Verificando-se a disseminação das fake news e da infodemia durante o período de infecção por covid-19, faz-se necessário utilizar ferramentas digitais seguras, como vídeos educativos institucionais, no intuito de informar melhor a população sobre os cuidados necessários sobre a doença e formas de atenuar o sofrimento psíquico.

Vários estudos realizados na pandemia têm apontado que as intervenções psicológicas online em tempos de emergência podem, portanto, ser fundamentais para aumentar a resiliência da população e identificar eventuais problemas psicológicos, de forma a implementar uma gestão atempada. Isso também é possível por meio de centros de aconselhamento psicológico (Savarese et al., 2020).

A utilização de vídeo como ferramenta educacional deve ter como objetivo potencializar o aprendizado e fomentar a construção do conhecimento. Isso implica que não basta inovar com a introdução dos meios de comunicação; também é importante contextualizar em ambientes de aprendizagem - virtuais ou não - e se adaptar à proposta pedagógica em que são utilizados. O vídeo pode ser usado de várias maneiras, seja (a) no modo vídeo-aula que transmite informações com suporte de vídeo para ilustrar o conteúdo como um programa monoconceito, focado em um conceito específico ou (b) como um programa motivador que sensibiliza o aluno a articular o conteúdo (Rodrigues et al., 2020).

Diante da exposição, verifica-se a necessidade de buscar analisar pela literatura nacional e internacional publicações de vídeos educativos sobre os cuidados em saúde mental para minimizar o sofrimento psíquico de populações mais vulneráveis, decorrentes do período pandêmico e pós-pandêmico. Os vídeos poderão disseminar uma mídia positiva sobre cuidados em saúde mental na pandemia para estudantes, profissionais e população em geral, em vários grupos etários e sociais, em diferentes contextos e cenários, como as unidades de saúde, escolas e outros espaços institucionais.

\section{Metodologia}

Realizou-se uma Revisão Integrativa (RI), a qual viabiliza a análise de pesquisas científicas de modo sistemático e amplo, favorecendo a caracterização e divulgação do conhecimento produzido (Cahú et al., 2011). Compõe-se de seis etapas, a saber: 1) definição do tema e elaboração da pergunta de pesquisa; 2) critérios de elegibilidade, inclusão e exclusão dos estudos; 3) levantamento dos estudos selecionados em formato de tabelas, considerando todas as características em comum; 4) categorização e análise crítica dos achados, identificando diferenças e conflitos; 5) interpretação dos resultados e 6) apresentação dos resultados, incluindo análise crítica dos achados e síntese da revisão (Souza et al., 2010).

Para a formulação da questão norteadora do estudo utilizou-se a pergunta PCC, ou seja, a estratégia PICO adaptada. Na adaptação, tal acrônimo significa P- população, C- conceito e C- contexto/desfecho. Assim, a estratégia foi formulada da seguinte maneira: P- profissionais da saúde; C- saúde mental; C- Covid-19.

Desta forma, estabeleceu-se, a seguinte questão norteadora: Quais as principais publicações de vídeos educativos sobre cuidados em saúde mental na pandemia encontradas na literatura nacional e internacional?

Após identificação dos Descritores em Ciências da Saúde (DeCS/MeSH), foi aplicada a estratégia de busca: "vídeo" AND "Covid-19" AND "mental health". As buscas dos estudos foram realizadas nos seguintes bancos de dados eletrônicos: Biblioteca Virtual em Saúde (BVS), Science Direct, Web of Science e CINAHL. O acesso às publicações ocorreu no mês de dezembro de 2021. Na elegibilidade dos estudos, conforme descritos na Figura 1, foram determinados como critérios de inclusão, estudos pesquisados no período de janeiro de 2020 a dezembro de 2021, limitando-se aos artigos com textos completos, open acess, publicados em todos os idiomas. Nos critérios de exclusão foram excluídos os estudos que não abordassem o tema da estratégia de busca. 
Após a realização da busca, foram encontrados 2.890 artigos que traziam informações sobre os descritores selecionados, distribuídos da seguinte forma: BVS (387), Science Direct (730), Web of Science (1697) e CINAHL (29). No entanto, depois da leitura dos títulos, resumos, íntegra dos artigos, foi necessário descartar aqueles que não faziam o cruzamento entre os três descritores, restando apenas 10 deles para análise.

$\mathrm{Na}$ terceira etapa, os dados obtidos foram agrupados de acordo com os enfoques dos títulos das publicações selecionadas para o estudo em uma tabela contendo as seguintes informações: título, ano de publicação, periódico e base de dado encontrada e principais resultados das publicações selecionadas.

A quarta etapa foi formulada com o início da avaliação dos estudos incluídos. Em sequência, na quinta etapa foi feita uma análise descritiva, que consiste na classificação dos elementos segundo suas semelhanças e por diferenciação, com posterior reagrupamento, em função de características comuns.

A sexta etapa consistiu na apresentação da revisão, com a análise e posterior síntese dos artigos que atenderam aos critérios de inclusão, utilizando-se quadros sinópticos.

\section{Resultados}

A busca inicial resultou em 2.890 estudos. Desse total, 2.880 foram excluídos por não se adequarem a estratégia de busca e/ou não responderem ao objeto de estudo. Portanto, o corpo de análise desta revisão integrativa foi composto por 10 estudos. A seleção foi realizada seguindo as diretrizes da Joanna Briggs Institute (JBI) como demonstrado na Figura 1 contendo o fluxograma PRISMA-ScR da (Munn et al., 2018; Jbi, 2020). 
Figura 1 - Fluxograma da seleção das publicações baseado no modelo PRISMA.

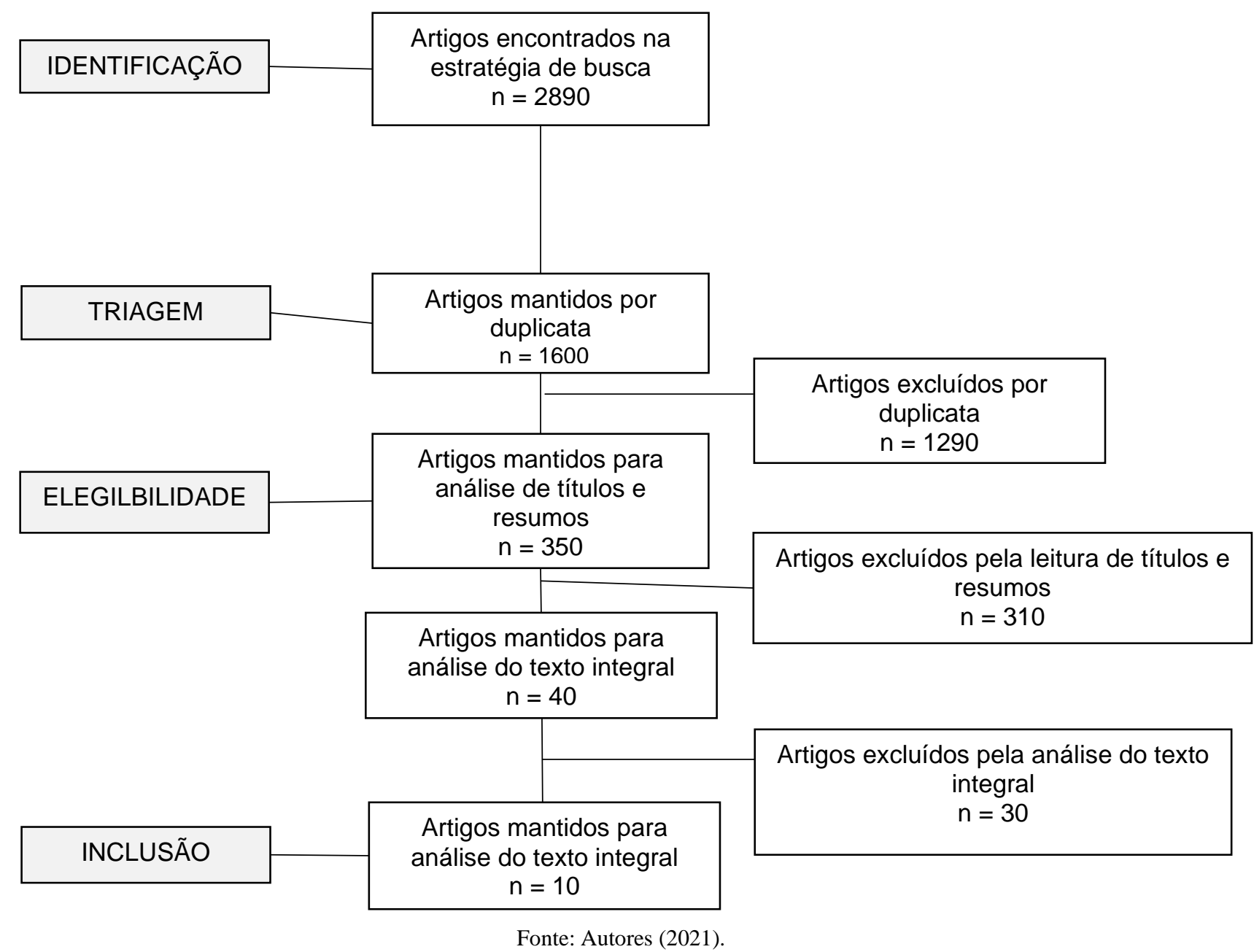

Os resultados encontrados foram distribuídos no Quadro 1, onde verifica-se que 9 artigos foram publicados em 2021 e apenas 1 em 2020. De todas as publicações avaliadas, 9 foram encontradas na base de dados Web of Science e apenas 1 no Science Direct, embora tenha havido publicações que estavam simultaneamente em várias bases de dados avaliadas. O quadro abaixo demonstra a síntese dos resultados de cada publicação selecionada. 
Quadro 1 - Caracterização dos estudos incluídos na revisão integrativa

\begin{tabular}{|c|c|c|c|}
\hline TÍTULO & $\begin{array}{c}\text { ANO DE } \\
\text { PUBLICAÇÃO }\end{array}$ & $\begin{array}{c}\text { PERIÓDICO } \\
\text { /BASE }\end{array}$ & PRINCIPAIS RESULTADOS \\
\hline $\begin{array}{l}\text { O impacto dos vídeos } \\
\text { de educação para a } \\
\text { saúde na saúde mental } \\
\text { e no comportamento do } \\
\text { público em geral } \\
\text { durante o COVID-19 }\end{array}$ & 2021 & $\begin{array}{c}\text { Global Health } \\
\text { Research and Policy/ } \\
\text { Web of Science }\end{array}$ & $\begin{array}{l}\text { Analisou as relações de mediação entre a exposição a vídeos de educação em saúde e } \\
\text { depressão/ansiedade/comportamentos relacionados à saúde. Análises de regressão } \\
\text { logística binária indicaram que as pessoas que assistiram aos vídeos de educação em } \\
\text { saúde eram mais propensas a usar máscaras (OR } 1,15, \mathrm{p}<0,001) \text {, desinfetar }(\mathrm{OR} 1,26, \mathrm{p} \\
<0,001 \text { ) e medir temperatura (OR } 1,37, \mathrm{p}<0,001) \text {. Com maior nível de crescimento pós- } \\
\text { traumático (PTG) ou suporte social percebido (PSS), as pessoas apresentaram menor } \\
\text { percentual de depressão (Para PSS, OR } 0,98, \mathrm{p}<0,001 \text {; Para PTG, OR } 0,98, \mathrm{p}<0,01) \text { e } \\
\text { ansiedade (Para PSS, OR } 0,98, \mathrm{p}<0,001 \text {. A exposição a vídeos de educação em saúde } \\
\text { pode melhorar a autopercepção do apoio social e o crescimento interno das pessoas e } \\
\text { ajudá-las a lidar com o impacto adverso das emergências de saúde pública com melhores } \\
\text { comportamentos relacionados à saúde mental e à saúde. }\end{array}$ \\
\hline $\begin{array}{l}\text { Isolamento social e } \\
\text { solidão de adultos mais } \\
\text { velhos em tempos de } \\
\text { pandemia do COVID- } \\
\text { 19: o uso de sites de } \\
\text { mídia social online e } \\
\text { chats de vídeo podem } \\
\text { ajudar a atenuar o } \\
\text { isolamento social e a } \\
\text { solidão? }\end{array}$ & 2021 & $\begin{array}{l}\text { Gerontology/ Web of } \\
\text { Science }\end{array}$ & $\begin{array}{l}\text { Com base em amostras representativas nacionalmente, apenas } 3 \text { estudos investigaram a } \\
\text { ligação entre sites de redes sociais on-line e solidão ou isolamento social entre idosos. } \\
\text { Enquanto um primeiro estudo se concentrou em vários desfechos (ou seja, conexão social } \\
\text { e isolamento social), um segundo estudo se concentrou na solidão e um terceiro estudo se } \\
\text { concentrou no isolamento social como medida de desfecho. Dados demonstraram que as } \\
\text { comunidades on-line são "lugares onde as pessoas podem se reunir e se envolver em } \\
\text { contato social, por exemplo, superar a solidão à noite". Portanto, ela conclui que esses } \\
\text { sites podem a superar sentimentos de solidão e isolamento. }\end{array}$ \\
\hline $\begin{array}{l}\text { Eficácia de uma } \\
\text { intervenção baseada em } \\
\text { vídeo na redução das } \\
\text { percepções de medo, } \\
\text { solidão e estigma } \\
\text { público relacionados ao } \\
\text { COVID-19: um ensaio } \\
\text { clínico randomizado }\end{array}$ & 2021 & $\begin{array}{l}\text { International Journal } \\
\text { of Public } \\
\text { Health/Web of } \\
\text { Science }\end{array}$ & $\begin{array}{l}\text { Durante o primeiro pico do surto de COVID-19 nos Estados Unidos, investigamos o } \\
\text { impacto de intervenções digitais para reduzir o medo, a solidão e o estigma público } \\
\text { relacionados à COVID-19. Intervenções baseadas em vídeo levam a reduções no medo e } \\
\text { estigma relacionados à COVID-19. Não foi encontrada diferença na atividade social } \\
\text { entre os grupos, potencialmente explicando a falta de eficácia na solidão. }\end{array}$ \\
\hline $\begin{array}{l}\text { Tele-reabilitação para } \\
\text { pessoas com demência } \\
\text { durante a pandemia } \\
\text { COVID-19: um estudo } \\
\text { de caso da Inglaterra }\end{array}$ & 2021 & $\begin{array}{l}\text { INTERNATIONAL } \\
\text { JOURNAL OF } \\
\text { ENVIRONMENTAL } \\
\text { RESEARCH AND } \\
\text { PUBLIC } \\
\text { HEALTH/WEB OF } \\
\quad \text { SCIENCE }\end{array}$ & $\begin{array}{l}\text { A entrega de vídeo funcionou melhor quando os participantes tinham um cuidador de } \\
\text { apoio e quando os terapeutas mostraram entusiasmo e tiveram um relacionamento } \\
\text { estabelecido com o cliente. Os benefícios incluíram eficiência de tempo das sessões, } \\
\text { aumento da motivação dos participantes, conscientização sobre demência dos cuidadores } \\
\text { e criatividade dos terapeutas. As limitações incluíram as habilidades e recursos de TI } \\
\text { ruins dos usuários. A pandemia de COVID-19 exigiu maneiras inovadoras de } \\
\text { proporcionar reabilitação. Este estudo apoia que as pessoas com demência podem usar a } \\
\text { tele-reabilitação, mas o sucesso depende de ter um cuidador e um terapeuta entusiasmado } \\
\text { e conhecido. }\end{array}$ \\
\hline $\begin{array}{l}\text { O uso de vídeo para } \\
\text { informação e educação } \\
\text { do paciente: uma } \\
\text { análise do escopo da } \\
\text { variabilidade e eficácia } \\
\text { das intervenções }\end{array}$ & 2021 & $\begin{array}{l}\text { Patient Education } \\
\text { and Counseling / } \\
\text { Science Direct }\end{array}$ & $\begin{array}{l}\text { Fornecer uma visão geral das intervenções em vídeo usadas para informação e educação } \\
\text { do paciente, e das ferramentas usadas para avaliar sua eficácia, a fim de considerar a } \\
\text { viabilidade do desenvolvimento de diretrizes genéricas e ferramentas de avaliação para o } \\
\text { uso de vídeo no atendimento ao paciente. Temas comuns emergem, notadamente o } \\
\text { objetivo de reduzir a ansiedade e a variedade de instrumentos projetados para medir isso. } \\
\text { A intervenção direcionada baseada em vídeo pode melhorar a experiência e os resultados } \\
\text { do paciente. }\end{array}$ \\
\hline $\begin{array}{l}\text { Covid-19: análise } \\
\text { métrica de vídeos e } \\
\text { canais de comunicação } \\
\text { no YouTube }\end{array}$ & 2020 & $\begin{array}{c}\text { El profesional de la } \\
\text { información / Web of } \\
\text { Science }\end{array}$ & $\begin{array}{l}\text { Os resultados mostram que o número de vídeos sobre a Covid-19 aumentou desde o } \\
\text { estabelecimento do estado de alarme na Espanha e, pouco a pouco, tem diminuído ao } \\
\text { longo do tempo, com certas reviravoltas ligadas a decisões políticas. Os vídeos alcançam, } \\
\text { em média, um grande número de visualizações, curtidas/desgostos e comentários, e } \\
\text { foram publicados principalmente pela mídia. Vídeos relacionados a blogs e } \\
\text { entretenimento são muito numerosos, mas com menos impacto. Os vídeos pertencentes } \\
\text { às categorias Educação e Ciência e Tecnologia são menores, mas com alto impacto, } \\
\text { especialmente em visualizações. Além disso, conclui-se que os critérios de } \\
\text { classificação do YouTube não são precisos o suficiente para serem usados em estudos } \\
\text { métricos informativos sem uma alta carga de trabalho na limpeza de dados. Da mesma } \\
\text { forma, a existência de canais que aplicam estratégias de posicionamento enganosas } \\
\text { dificulta a realização desse tipo de estudo. }\end{array}$ \\
\hline $\begin{array}{l}\text { Intervenção cognitivo- } \\
\text { comportamental } \\
\text { baseada em vídeo para } \\
\text { ansiedade COVID-19: } \\
\text { um ensaio clínico } \\
\text { randomizado }\end{array}$ & 2021 & $\begin{array}{c}\text { Trends Psychiatry } \\
\text { Psychother/ Web of } \\
\text { Science }\end{array}$ & $\begin{array}{l}\text { O objetivo deste estudo foi investigar a eficácia de uma intervenção cognitivo- } \\
\text { comportamental baseada em vídeo para reduzir a ansiedade por COVID-19. Oitenta por } \\
\text { cento relataram que a intervenção baseada em vídeo foi uma alternativa benéfica à } \\
\text { intervenção terapêutica presencial tradicional. Na avaliação pós-tratamento, o grupo de } \\
\text { intervenção cognitivo-comportamental baseada em vídeo mostrou uma redução } \\
\text { significativa na ansiedade COVID-19, ansiedade em saúde, sensibilidade à ansiedade e } \\
\text { amplificação somatossensorial quando comparado ao grupo controle na lista de espera. } \\
\text { Este estudo sugere que intervenções cognitivo-comportamentais baseadas em vídeo } \\
\text { podem ser um método acessível, viável e eficaz para reduzir a ansiedade durante uma } \\
\text { pandemia de grande escala. }\end{array}$ \\
\hline $\begin{array}{lr}\text { Comunicação } & \text { baseada } \\
\text { em vídeo e sua } \\
\text { associação } r \text { com } \\
\text { solidão, saúde mental e } \\
\text { qualidade de vida entre }\end{array}$ & 2021 & $\begin{array}{l}\text { International Journal } \\
\text { of Environmental } \\
\text { Research and Public } \\
\text { Health/ Web of } \\
\text { Science }\end{array}$ & $\begin{array}{l}\text { O objetivo do estudo foi examinar o uso da comunicação baseada em vídeo e sua } \\
\text { associação com solidão, saúde mental e qualidade de vida em idosos ( } 60 \text { a } 69 \text { anos versus } \\
\text { mais de } 70 \text { anos) durante a pandemia de COVID-19. Análises de regressão múltipla } \\
\text { foram realizadas para examinar associações entre o uso de ferramentas de comunicação } \\
\text { baseadas em vídeo e solidão, saúde mental e qualidade de vida dentro das faixas etárias, }\end{array}$ \\
\hline
\end{tabular}




\begin{tabular}{|c|c|c|c|}
\hline $\begin{array}{l}\text { idosos durante o surto } \\
\text { de COVID-19 }\end{array}$ & & & $\begin{array}{l}\text { enquanto ajustando por variáveis sociodemográficas. Instrumentos de comunicação } \\
\text { baseados em vídeo foram mais usados entre participantes de } 60 \text { a } 69 \text { anos }(60,1 \%) \text {, em } \\
\text { comparação com participantes com } 70 \text { anos ou mais }(51,8 \%, p<0,05) \text {. Ajustando para } \\
\text { todas as variáveis, o uso de comunicação baseada em vídeo foi associado a menos } \\
\text { solidão }(\beta=-0,12, p<0,01) \text { e maior qualidade de vida }(\beta=0,14, p<0,01) \text { entre os } \\
\text { participantes de } 60 \text { a } 69 \text { anos, enquanto não foram observadas associações para os } \\
\text { participantes da faixa etária mais avançada. O uso de ferramentas de comunicação por } \\
\text { vídeo foi, portanto, associado a resultados psicológicos favoráveis entre os participantes } \\
\text { na casa dos sessenta anos, mas não entre os participantes da faixa etária mais velha. Os } \\
\text { resultados do estudo apoiam a noção de que a idade pode influenciar a associação entre o } \\
\text { uso de ferramentas de comunicação por vídeo e resultados psicológicos entre pessoas } \\
\text { idosas. }\end{array}$ \\
\hline $\begin{array}{l}\text { Aumento do uso de } \\
\text { ferramentas } \\
\text { tecnologias digitais de } \\
\text { saúde mental nos } \\
\text { Estados Unidos durante } \\
\text { a pandemia COVID- } \\
\text { 19: estudo de pesquisa }\end{array}$ & 2021 & $\begin{array}{c}\text { Journal of medical } \\
\text { internet research/ } \\
\text { Web of Science }\end{array}$ & $\begin{array}{l}\text { O objetivo deste estudo foi examinar sistematicamente se houve um aumento relacionado } \\
\text { à pandemia de COVID-19 no uso autorreferido de ferramentas digitais de saúde mental e } \\
\text { outras tecnologias para gerenciar a saúde mental. Os resultados sugeriram o aumento do } \\
\text { uso de ferramentas digitais de saúde mental e outras tecnologias ao longo do tempo } \\
\text { durante os estágios iniciais da pandemia de COVID-19. Como tal, é urgentemente } \\
\text { necessário um esforço adicional para considerar a qualidade desses produtos, seja } \\
\text { garantindo que os usuários tenham acesso a tecnologias baseadas em evidências e } \\
\text { baseadas em evidências e/ou fornecendo-lhes as habilidades para tomar decisões } \\
\text { informadas sobre sua potencial eficácia. }\end{array}$ \\
\hline $\begin{array}{l}\text { Serviços remotos de } \\
\text { saúde mental: uma } \\
\text { pesquisa de métodos } \\
\text { mistos e um estudo de } \\
\text { entrevista sobre o uso, } \\
\text { valor, benefícios e } \\
\text { desafios de um serviço } \\
\text { nacional de consultoria } \\
\text { de vídeo no NHS } \\
\text { Wales, Reino Unido }\end{array}$ & 2021 & $\begin{array}{l}\text { BMJ Open/ Web of } \\
\text { Science }\end{array}$ & $\begin{array}{l}\text { Um total de } 3561 \text { participantes forneceram dados específicos de saúde mental. Esses } \\
\text { dados e seus achados demonstram que a prestação remota de serviços de saúde mental, } \\
\text { através do método da Consultoria Remota é altamente satisfatória, bem aceita e } \\
\text { clinicamente adequada para muitos pacientes, e oferece uma série de benefícios para } \\
\text { pacientes e clínicos. Curiosamente, os clínicos que trabalham em 'casa' classificaram a } \\
\text { Consultoria Remota de forma mais positiva em comparação com aqueles em sua 'base } \\
\text { clínica'. Após a adoção de } 1 \text { ano, os serviços remotos de saúde mental no País de Gales, } \\
\text { Reino Unido, demonstraram que a Consultoria Remota é possível tanto do ponto de vista } \\
\text { técnico quanto comportamental. Avançando, sugerimos líderes clínicos e apoio do } \\
\text { governo para sustentar essa abordagem 'por padrão'. }\end{array}$ \\
\hline
\end{tabular}

Fonte: Autores (2021).

\section{Discussão}

Refletindo o aumento nos casos e mortes de Covid-19, houve também um aumento no sofrimento mental da população. Dados dos Estados Unidos apontam que adultos avaliados como parte de uma pesquisa nacionalmente representativa em abril e maio de 2020 tinham mais de três vezes mais chances de fazer triagem positiva para transtornos depressivos, transtornos de ansiedade ou ambos, em relação a uma amostra comparável em 2019.

Na mesma linha, os Centros de Controle e Prevenção de Doenças relataram níveis significativamente elevados de condições adversas de saúde mental, uso de substâncias e ideação suicida resultante da pandemia Covid-19, com essas condições de saúde mental afetando desproporcionalmente populações específicas, como jovens adultos, hispânicos, negros, trabalhadores essenciais, cuidadores não remunerados de adultos e aqueles que recebem tratamento para condições psiquiátricas preexistentes (Sorkin et al., 2021).

Semelhante a doenças epidêmicas anteriores, Covid-19 fez com que membros de muitas comunidades se sentissem ansiosos e frustrados. Intervenções cognitivo-comportamentais têm se mostrado bastante eficazes na redução da ansiedade, especialmente na redução da ansiedade relacionada à saúde (Shabahang et al., 2021).

Em uma época em que indivíduos em todo o mundo estão vivenciando a propagação de uma pandemia, é fundamental avaliar o potencial aumento dos fenômenos de medo, estigma público e de solidão percebida, pois estes podem afetar o comportamento, mental e saúde física. As intervenções de vídeo demonstraram ser eficazes na mitigação do estigma da saúde mental. No entanto, até onde sabemos, as evidências da eficácia das intervenções baseadas em vídeo para mitigar o medo, a solidão e o estigma relacionados ao Covid-19 são muito limitadas (Valeri et al., 2021).

As ferramentas de comunicação atuais baseadas em vídeo usam uma conexão com a Internet para permitir que os usuários se comuniquem em tempo real e se vejam enquanto o fazem. Nos últimos anos, várias dessas ferramentas também foram desenvolvidas para permitir o compartilhamento digital de materiais entre as pessoas que participam da chamada. Assim, as ferramentas de comunicação baseadas em vídeo podem aumentar muito as possibilidades de troca, bem como a 
experiência geral de comunicação, em comparação com chamadas telefônicas regulares, principalmente em épocas de pandemia (Bonsaksen et al. 2021).

É importante avaliar a educação em saúde baseada em vídeo que promova comportamentos de saúde durante a pandemia de Covid-19. Os vídeos de educação para a saúde que incentivam o público a manter a mente calma, a não ter medo ou pânico, ensinar maneiras de aliviar emoções negativas, ensinar as pessoas como se comportar de forma adequada e enfatizar os benefícios da adoção de comportamentos relacionados à saúde para os indivíduos e a sociedade (Yang et al., 2021).

Até o momento, poucos estudos investigaram a associação entre o uso de chats de vídeo ou mídias sociais online e o isolamento social ou solidão entre idosos. Uma solução possível forma para lidar com essa situação e ficar em contato com outras pessoas é usar chats de vídeo (por exemplo, Skype) ou mídia social online (por exemplo, Facebook). Essas soluções podem, pelo menos temporariamente, substituir o contato físico (por exemplo, com amigos, conhecidos, filhos ou netos) em tempos de pandemia de Covid-19. Parece plausível que essas possibilidades possam reduzir os sentimentos de isolamento social ou solidão (por exemplo, ao ver os netos no monitor ou smartphone).

Além disso, indivíduos mais velhos que usam chats de vídeo ou sites de mídia social online para ficar em contato com a família e amigos podem pensar que estão em melhor situação do que quando não usaram tais possibilidades técnicas durante o auto-isolamento (comparação intraindividual). Assim, eles podem apreciar muito essas soluções técnicas para superar os sentimentos de isolamento e solidão (Hajek; Konig, 2021).

As evidências também estão aumentando para a eficácia da tele-reabilitação, em comparação com a reabilitação face a face. O potencial de custo e eficiência de tempo da tele-reabilitação foi observado. Viajar por longas distâncias (onde os serviços cobrem grandes áreas de captação) ou por um longo tempo (no caso de conurbações metropolitanas) para sessões de reabilitação face a face exige muitos recursos (Lorito et al., 2021).

Ferramentas terapêuticas baseadas em vídeo podem oferecer oportunidades de fornecer intervenções psicológicas eficazes para a ansiedade Covid-19 em grandes populações. Nossos resultados apóiam a aplicabilidade da intervenção cognitivo-comportamental baseada em vídeo na ansiedade Covid-19. Em outras palavras, uma intervenção cognitivocomportamental baseada em vídeo de autoajuda é um meio eficaz de aliviar a ansiedade Covid-19, ansiedade de saúde, sensibilidade à ansiedade e amplificação somatossensorial (Shabahang et al., 2021).

Refletindo o aumento nos casos e mortes de Covid-19, houve também um aumento no sofrimento mental da população. Dados dos Estados Unidos apontam que adultos avaliados como parte de uma pesquisa nacionalmente representativa em abril e maio de 2020 tinham mais de três vezes mais chances de fazer triagem positiva para transtornos depressivos, transtornos de ansiedade ou ambos, em relação a uma amostra comparável em 2019.

Na mesma linha, os Centros de Controle e Prevenção de Doenças relataram níveis significativamente elevados de condições adversas de saúde mental, uso de substâncias e ideação suicida resultante da pandemia Covid-19, com essas condições de saúde mental afetando desproporcionalmente populações específicas, como jovens adultos, hispânicos, negros, trabalhadores essenciais, cuidadores não remunerados de adultos e aqueles que recebem tratamento para condições psiquiátricas preexistentes (Sorkin et al., 2021).

Semelhante a doenças epidêmicas anteriores, Covid-19 fez com que membros de muitas comunidades se sentissem ansiosos e frustrados. Intervenções cognitivo-comportamentais têm se mostrado bastante eficazes na redução da ansiedade, especialmente na redução da ansiedade relacionada à saúde (Shabahang et al., 2021).

Em uma época em que indivíduos em todo o mundo estão vivenciando a propagação de uma pandemia, é fundamental avaliar o potencial aumento dos fenômenos de medo, estigma público e de solidão percebida, pois estes podem afetar o comportamento, mental e saúde física. As intervenções de vídeo demonstraram ser eficazes na mitigação do estigma da saúde 
mental. No entanto, até onde sabemos, as evidências da eficácia das intervenções baseadas em vídeo para mitigar o medo, a solidão e o estigma relacionados ao Covid-19 são muito limitadas (Valeri et al., 2021).

As ferramentas de comunicação atuais baseadas em vídeo usam uma conexão com a Internet para permitir que os usuários se comuniquem em tempo real e se vejam enquanto o fazem. Nos últimos anos, várias dessas ferramentas também foram desenvolvidas para permitir o compartilhamento digital de materiais entre as pessoas que participam da chamada. Assim, as ferramentas de comunicação baseadas em vídeo podem aumentar muito as possibilidades de troca, bem como a experiência geral de comunicação, em comparação com chamadas telefônicas regulares, principalmente em épocas de pandemia (Bonsaksen et al. 2021).

É importante avaliar a educação em saúde baseada em vídeo que promova comportamentos de saúde durante a pandemia de Covid-19. Os vídeos de educação para a saúde que incentivam o público a manter a mente calma, a não ter medo ou pânico, ensinar maneiras de aliviar emoções negativas, ensinar as pessoas como se comportar de forma adequada e enfatizar os benefícios da adoção de comportamentos relacionados à saúde para os indivíduos e a sociedade (Yang et al., 2021).

Até o momento, poucos estudos investigaram a associação entre o uso de chats de vídeo ou mídias sociais online e o isolamento social ou solidão entre idosos. Uma solução possível forma para lidar com essa situação e ficar em contato com outras pessoas é usar chats de vídeo (por exemplo, Skype) ou mídia social online (por exemplo, Facebook). Essas soluções podem, pelo menos temporariamente, substituir o contato físico (por exemplo, com amigos, conhecidos, filhos ou netos) em tempos de pandemia de Covid-19. Parece plausível que essas possibilidades possam reduzir os sentimentos de isolamento social ou solidão (por exemplo, ao ver os netos no monitor ou smartphone).

Além disso, indivíduos mais velhos que usam chats de vídeo ou sites de mídia social online para ficar em contato com a família e amigos podem pensar que estão em melhor situação do que quando não usaram tais possibilidades técnicas durante o auto-isolamento (comparação intraindividual). Assim, eles podem apreciar muito essas soluções técnicas para superar os sentimentos de isolamento e solidão (Hajek \& Konig, 2021).

As evidências também estão aumentando para a eficácia da tele-reabilitação, em comparação com a reabilitação face a face. O potencial de custo e eficiência de tempo da tele-reabilitação foi observado. Viajar por longas distâncias (onde os serviços cobrem grandes áreas de captação) ou por um longo tempo (no caso de conurbações metropolitanas) para sessões de reabilitação face a face exige muitos recursos (Lorito et al., 2021).

Ferramentas terapêuticas baseadas em vídeo podem oferecer oportunidades de fornecer intervenções psicológicas eficazes para a ansiedade Covid-19 em grandes populações. Nossos resultados apóiam a aplicabilidade da intervenção cognitivo-comportamental baseada em vídeo na ansiedade Covid-19. Em outras palavras, uma intervenção cognitivocomportamental baseada em vídeo de autoajuda é um meio eficaz de aliviar a ansiedade Covid-19, ansiedade de saúde, sensibilidade à ansiedade e amplificação somatossensorial (Shabahang et al., 2021).

\section{Considerações Finais}

Após uma ampla revisão de publicações sobre o uso de vídeo para atenuar o sofrimento psíquico gerado pela pandemia do covid-19, foi possível identificar que essa ferramenta digital promove apoio social e autoajuda para pessoas de diferentes faixas etárias. Os vídeos educativos minimizam a depressão e a ansiedade gerada pelo medo de contrair a infecção, reduz o sentimento de solidão e serve como terapia em saúde mental, além de ser viável, eficaz e acessível para a maioria da população. 


\section{Referências}

Amarante, P., et al. (2020). Enfrentamento do sofrimento psíquico na pandemia: diálogos sobre o acolhimento e a saúde mental em territórios vulnerabilizados. IdeiaSUS/Fiocruz.

Cahu, G. P. R., et al. (2011). Produção científica em periódicos online acerca da prática do assédio moral: uma revisão integrativa. Rev. Gaúcha Enferm. 32(3), 611-9.

Chatterjee A, Strong G, Meinert E, Milne-Ives M, Halkes M, \& Wyatt-Haines E. (2021). The use of video for patient information and education: A scoping review of the variability and effectiveness of interventions. Patient Educ Couns. 104(9):2189-2199. 10.1016/j.pec.2021.02.009.

de Figueiredo, C. S., Sandre, P. C., Portugal, L. C. L., Mázala-de-Oliveira, T., da Silva Chagas, L., Raony, Í., Ferreira, E. S., Giestal-de-Araujo, E., Dos Santos, A. A. \& Bomfim, P. O. (2021). COVID-19 pandemic impact on children and adolescents' mental health: Biological, environmental, and social factors. Prog Neuropsychopharmacol Biol Psychiatry. 106:110171. 10.1016/j.pnpbp.2020.110171“.

Di Lorito C, Duff C, Rogers C, Tuxworth J, Bell J, Fothergill R, Wilkinson L, Bosco A, Howe L, O'Brien R, Godfrey M, Dunlop M, van der Wardt V, Booth V, Logan P, Cowley A, \& Harwood, R. H (2021). Tele-Rehabilitation for People with Dementia during the COVID-19 Pandemic: A Case-Study from England. Int J Environ Res Public Health. 18(4):1717. 10.3390/ijerph18041717.

Grolli, R. E., Mingoti, M. E. D., \& Bertollo, (2021). A.G. et al. Impact of COVID-19 in the Mental Health in Elderly: Psychological and Biological Updates. Mol Neurobiol 58, 1905-1916.

Hajek, A., \& König, H. H. (2021). Social Isolation and Loneliness of Older Adults in Times of the COVID-19 Pandemic: Can Use of Online Social Media Sites and Video Chats Assist in Mitigating Social Isolation and Loneliness? Gerontology. 67(1):121-124. 10.1159/000512793.

Institute TJB (2020). Joanna Briggs Institute Reviewers' Manual: 2020 Edition. https://wiki.jbi.global/display/MANUAL/11.2+Development+of+a+scopin g+review+protoco

Johns G, Burhouse A, Tan J, John O, Khalil S, Williams J, Whistance B, Ogonovsky M, \& Ahuja A. (2021). Serviços remotos de saúde mental: uma pesquisa de métodos mistos e estudo de entrevista sobre o uso, valor, benefícios e desafios de um serviço nacional de consultoria em vídeo no NHS Wales, Reino Unido. BMJ Aberto. 11(9):e053014. 10.1136/bmjopen-2021-053014.

"Li, B. Y. \& Ho, R. T. H. (2019). Unveiling the Unspeakable: Integrating Video Elicitation Focus Group Interviews and Participatory Video in an Action Research Project on Dementia Care Development. International Journal of Qualitative Methods. 10.1177/160940691983056.

Munn Z, Peters MD, Stern C, Tufanaru C, McArthur A, \& Aromataris E. (2021). Systematic review or scoping review? Guidance for authors when choosing between a systematic or scoping review approach. BMC Med Res Methodol. 2018;18(1):143.

Nabuco, G., Oliveira, M. H. P. P., \& Afonso, M. P. D. (2020). O impacto da pandemia pela COVID-19 na saúde mental: qual é o papel da Atenção Primária à Saúde? Rev Bras Med Fam Comunidade. 15(42):2532. https://doi.org/10.5712/rbmfc15(42)2532.

Orduña-Malea, E.; Font-Julián, C. I.; \& Ontalba-Ruipérez, J-A. (2020). "Covid-19: análisis métrico de vídeos y canales de comunicación en YouTube”. El profesional de la información, 29(4), e290401. https://doi.org/10.3145/epi.2020.jul.01

Savarese, G., Curcio, L., D'Elia, D., Fasano, O., \& Pecoraro, N. (2020). Online University Counselling Services and Psychological Problems among Italian Students in Lockdown Due to Covid-19. Healthcare (Basel). 8(4):440. 10.3390/healthcare8040440.

Shabahang, R., Aruguete, M. S., \& McCutcheon, L. (2021). Video-based cognitive-behavioral intervention for COVID-19 anxiety: a randomized controlled trial. Trends Psychiatry Psychother. 43(2):141-150. 10.47626/2237-6089-2020-0056.

Sorkin, D. H., Janio, E. A., Eikey, E. V., Schneider, M., Davis, K., Schueller, S. M., Stadnick, N. A., Zheng, K., Neary, M., Safani, D., \& Mukamel, D. B. (2021). Aumento no Uso de Ferramentas e Tecnologias Digitais de Saúde Mental nos Estados Unidos Durante a Pandemia da COVID-19: Estudo de Pesquisa. J Med Internet Res. 23(4):e26994. 10.2196/26994.

Valeri, L. et al. (2021). "Effectiveness of a Video-Based Intervention on Reducing Perceptions of Fear, Loneliness, and Public Stigma Related to COVID-19: A Randomized Controlled Trial.” International journal of public health vol. 66 1604164. 10.3389/ijph.2021.1604164.

Video-Based Communication and Its Association with Loneliness. (2021). Mental Health and Quality of Life among Older People during the COVID-19 Outbreak. Int J Environ Res Public Health. 18 (12):6284. 10.3390/ijerph18126284.

Yang, Q. et al. (2021). "The impact of health education videos on general public's mental health and behavior during COVID-19." Global health research and policy $6,137.10 .1186 / \mathrm{s} 41256-021-00211-5$. 